Supporting Information for:

\title{
Differently Linked Perylene Bisimide Dimers with Various Twisting and Phase Structures for Nonfullerene All-Small Molecule Organic \\ Solar Cells
}

Rui Xin, ${ }^{1}$ Cheng Zeng, ${ }^{2}$ Dong Meng, ${ }^{2}$ Zhongjie Ren, ${ }^{3 *}$ Wei Jiang, ${ }^{2 *}$ Zhaohui Wang, ${ }^{4}$ and Shouke $\operatorname{Yan}^{1,3 *}$

${ }^{1}$ Key Laboratory of Rubber-Plastics, Ministry of Education, Qingdao University of Science \& Technology, Qingdao 266042, China

${ }^{2}$ Key Laboratory of Organic Solids, Beijing National Laboratory for Molecular Sciences, Institute of Chemistry, Chinese Academy of Sciences, Beijing 100190, China

${ }^{3}$ State Key Laboratory of Chemical Resource Engineering, Beijing University of Chemical Technology, Beijing, 100029, China

4 Key Laboratory of Organic Optoelectronics and Molecular Engineering, Department of Chemistry, Tsinghua University, Beijing 100084, P. R. China.

*E-mail: renzj@mail.buct.edu.cn; jiangwei@iccas.ac.cn; skyan@qust.edu.cn.

\section{Table of Contents}

Table S1 The device performance parameters for the solar cells with different D/A ratios for DR3TBDTT:B-SdiPBI, DR3TBDTT:O-SdiPBI and DR3TBDTT:H-SdiPBI blends S2

Figure. S1 $J^{0.5}$ vs V plots for the hole-only fabricated from DR3TBDTT: B-SdiPBI blend film (a) without SVA and (b) with SVA, DR3TBDTT: O-SdiPBI blend film (c) without SVA and (d) with SVA, DR3TBDTT: H-SdiPBI blend film (e) without SVA and (f) with SVA. .S3

Figure. S2 $J^{0.5}$ vs V plots for the electron-only fabricated from DR3TBDTT: B-SdiPBI blend film (a) without SVA and (b) with SVA, DR3TBDTT: O-SdiPBI blend film (c) without SVA and (d) with SVA, DR3TBDTT: H-SdiPBI blend film (e) without SVA and (f) with SVA. S4

Figure. S3 TEM images (scale bar $=0.2 \mu \mathrm{m}$ ) of DR3TBDTT: B-SdiPBI blend film (a) without SVA and (b) with SVA, DR3TBDTT: O-SdiPBI blend film (c) without SVA and (d) with SVA, DR3TBDTT: H-SdiPBI blend film (e) without SVA and (f) with SVA S5

Figure. S4 2D GIWAXS patterns for DR3TBDTT neat film (a,b), B-SdiPBI neat film (c,d), O-SdiPBI neat film (e,f) and H-SdiPBI neat film (g,h) without (a,c,e,g) and with (b,d,f,h) SVA treatment. .S6 
Table S1 The device performance parameters for the solar cells with different D/A ratios for DR3TBDTT:B-SdiPBI, DR3TBDTT:O-SdiPBI and DR3TBDTT:H-SdiPBI blend films.

\begin{tabular}{cccccc}
\hline Blends & $\begin{array}{c}\text { D/A } \\
\text { Ratios } \\
(\mathbf{w} / \mathbf{w})\end{array}$ & $\begin{array}{c}\text { Voc } \\
(\mathbf{V})\end{array}$ & $\begin{array}{c}\boldsymbol{J}_{\mathbf{S C}} \\
\left(\mathbf{m A} / \mathbf{c m}^{2}\right)\end{array}$ & $\begin{array}{c}\boldsymbol{F F} \\
(\boldsymbol{\%})\end{array}$ & $\begin{array}{c}\boldsymbol{P C E} \\
(\boldsymbol{\%})\end{array}$ \\
\hline & $1: 2$ & 0.91 & 1.45 & 28.85 & 0.38 \\
DR3TBDTT:B-SdiPBI & $1: 1$ & 0.93 & 2.81 & 29.83 & 0.78 \\
& $2: 1$ & 0.92 & 2.42 & 26.67 & 0.59 \\
\hline DR3TBDTT:O-SdiPBI & $1: 1$ & 0.93 & 5.87 & 34.16 & 1.86 \\
& $2: 1$ & 0.89 & 4.32 & 35.21 & 1.37 \\
\hline & $1: 2$ & 0.80 & 5.59 & 34.37 & 1.54 \\
& $1: 1$ & 0.88 & 5.82 & 44.65 & 2.30 \\
\hline & $2: 1$ & 0.88 & 7.26 & 47.48 & 3.05 \\
\hline
\end{tabular}



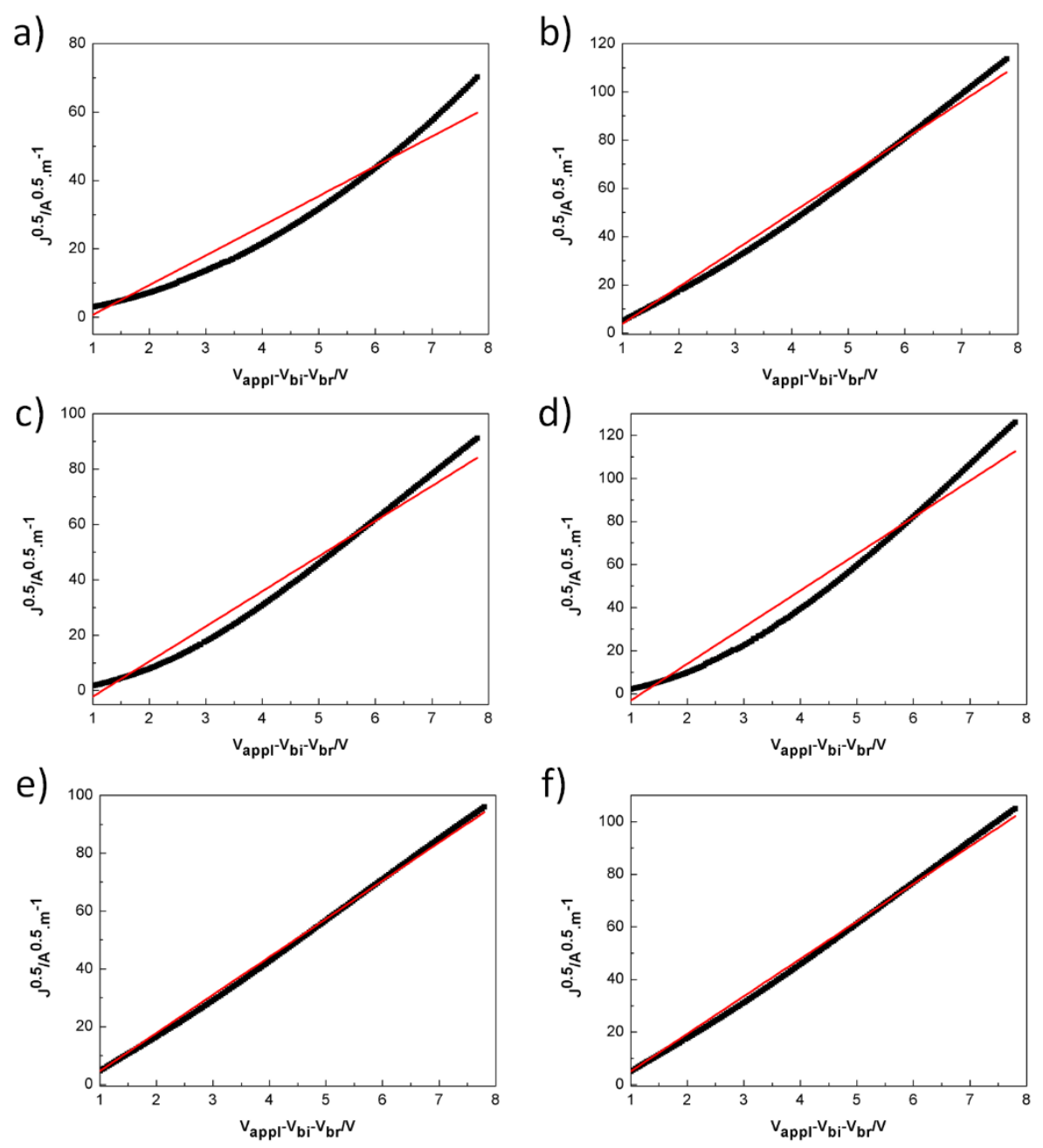

Figure. S1 $J^{0.5}$ vs V plots for the hole-only fabricated from DR3TBDTT: B-SdiPBI blend film (a) without SVA and (b) with SVA, DR3TBDTT: O-SdiPBI blend film (c) without SVA and (d) with SVA, DR3TBDTT: H-SdiPBI blend film (e) without SVA and (f) with SVA. 
a)

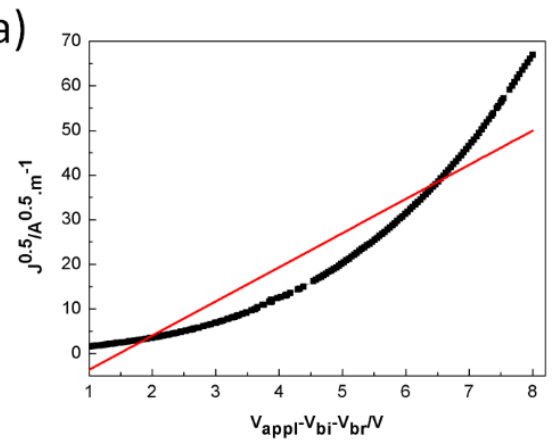

c)

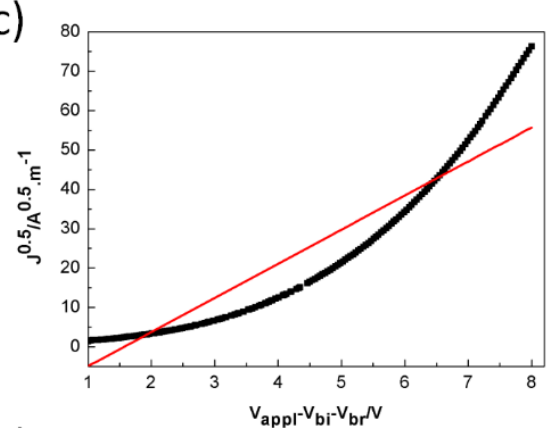

e)

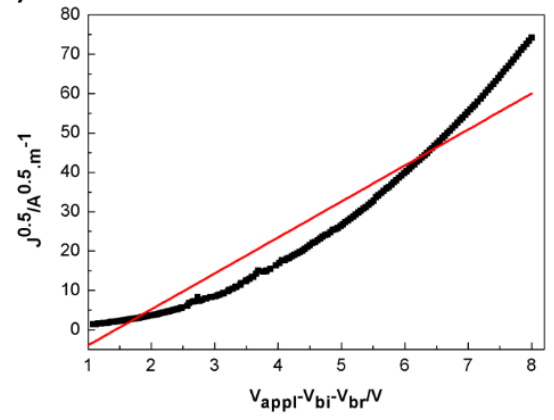

b)

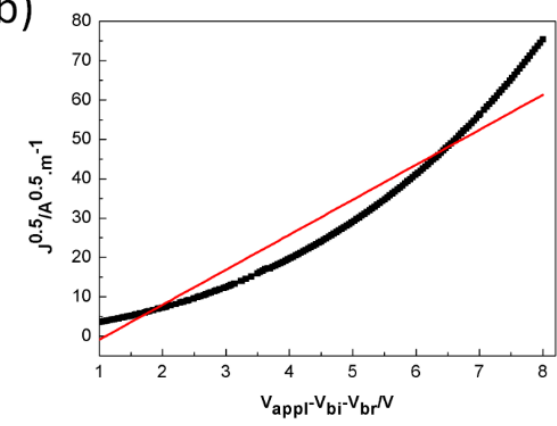

d)

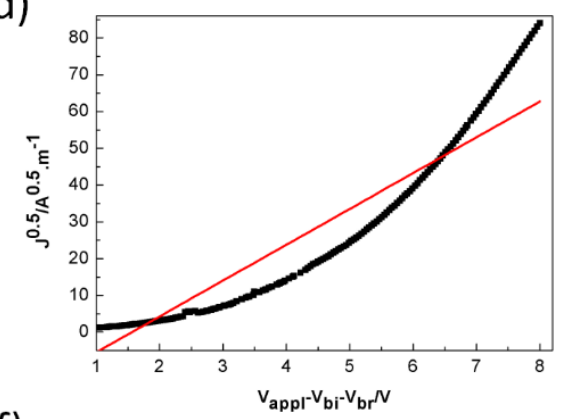

f)

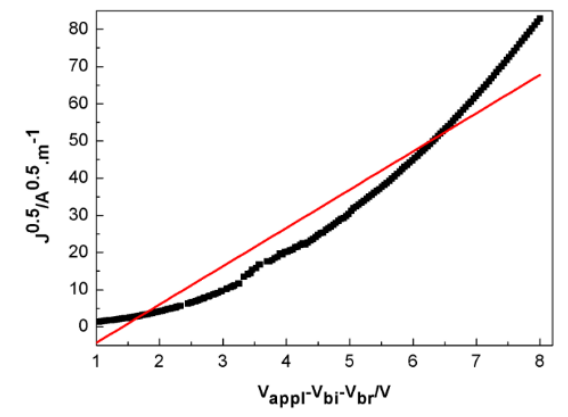

Figure. S2 $J^{0.5}$ vs V plots for the electron-only fabricated from DR3TBDTT: B-SdiPBI blend film (a) without SVA and (b) with SVA, DR3TBDTT: O-SdiPBI blend film (c) without SVA and (d) with SVA, DR3TBDTT: H-SdiPBI blend film (e) without SVA and (f) with SVA. 

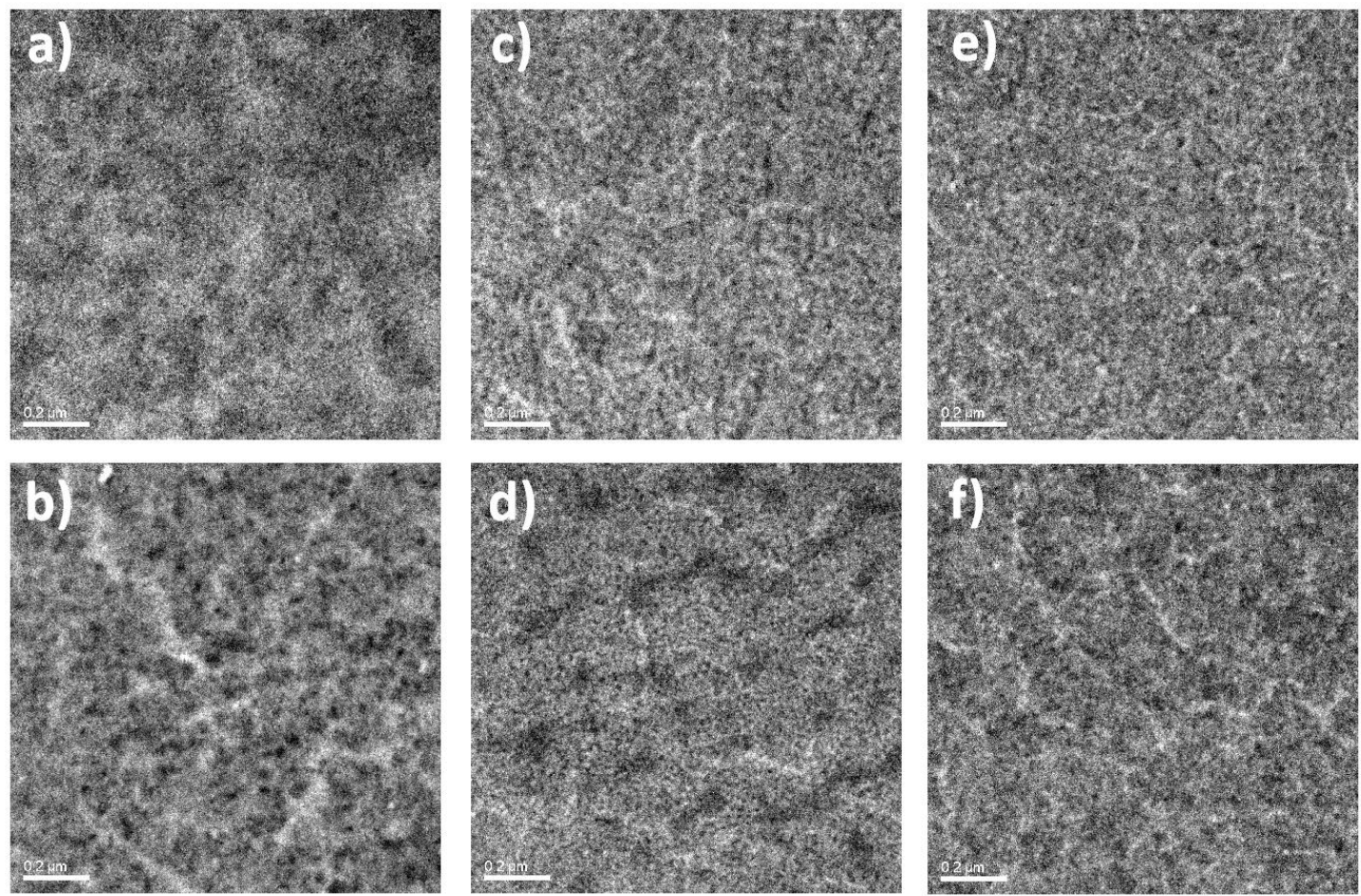

Figure. S3 TEM images (scale bar $=0.2 \mu \mathrm{m}$ ) of DR3TBDTT: B-SdiPBI blend film (a) without SVA and (b) with SVA, DR3TBDTT: O-SdiPBI blend film (c) without SVA and (d) with SVA, DR3TBDTT: H-SdiPBI blend film (e) without SVA and (f) with SVA. 


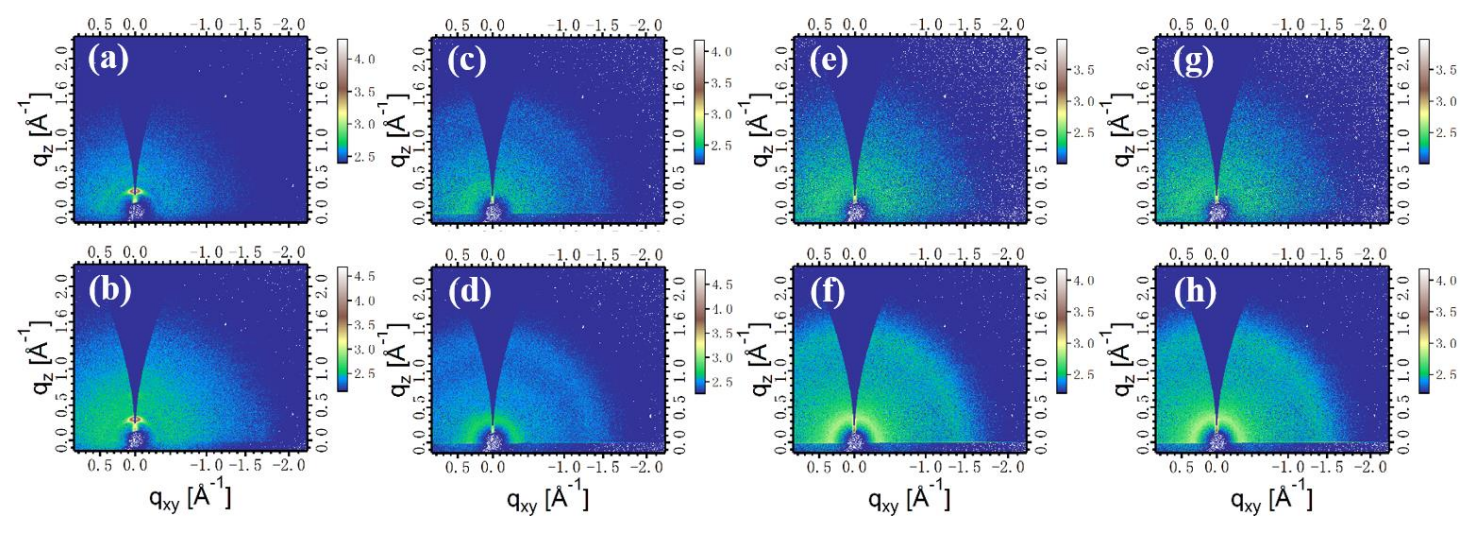

Figure. S4 2D GIWAXS patterns for DR3TBDTT neat film (a,b), B-SdiPBI neat film (c,d), O-SdiPBI neat film (e,f) and H-SdiPBI neat film (g,h) without (a,c,e,g) and with (b,d,f,h) SVA treatment. 